\title{
Sinovitis de la segunda articulación metatarsofalángica: actualización
}

\author{
Second Metatarsophalangeal joint Synovitis: Update
}

\section{Rubén SÁnchez Gómez ${ }^{1}$, Ruth González GutiérRez ${ }^{2}$, Daniel LóPez LóPez ${ }^{3}$, Marta Losa Iglesias ${ }^{4}$, Alfredo Soriano Medrano ${ }^{1}$, Ángel Morales Ponce ${ }^{1}$}

\footnotetext{
${ }^{1}$ Diplomado en Podología. Doctor por la Universidad Rey Juan Carlos de Madrid. Profesor del Máster Universitario de Investigación en Podología. Universidad Rey Juan Carlos de Madrid. España. rusago_@hotmail.com; soriano.alfredo@gmail.com; clinicatorrijos@gmail.com

${ }^{2}$ Diplomada en Podología. Mapfre-Clinicas. Madrid. España. monicaruth81@hotmail.com

${ }^{3}$ Diplomado en Podología. Doctor por la Universidad de La Coruña. Profesor del Máster Universitario de Investigación en Podología. Universidad Rey Juan Carlos de Madrid. España. daniellopez@udc.es

${ }^{4}$ Diplomada en Podología. Doctora por la Universidad de Oviedo. Profesor del Máster Universitario de Investigación en Podología. Universidad Rey Juan Carlos de Madrid. España. marta.losa@urjc.es
}

\section{Correspondencia: \\ Rubén Sánchez Gómez \\ C/ Santa Julia N ${ }^{\circ} 28$ p $2^{\circ} \mathrm{A}$ \\ E-28053 Madrid}

Fecha de recepción: 10 de marzo de 2013

Fecha de aceptación: 19 de septiembre de 2013

Los autores declaran no tener ningún tipo de interés económico o comercial.

\section{RESUMEN}

En las consultas de podología es común la existencia de numerosos procesos patológicos que desarrollan episodios inflamatorios en la cápsula articular de las articulaciones metatarsofalángicas (AMTF) y de sus estructuras vecinas como el plato glenoideo o los ligamentos colaterales, pero no son reconocidas claramente. Diferentes autores lo han explicado como el compromiso articular generado por un proceso de inestabilidad de etiología diversa y que evoluciona en diferentes estadíos, cada uno de los cuales posee diferentes grados de afectación a nivel de esa articulación. Esta patología se localiza en la articulación metatarsofalángica del segundo dedo con mayor prevalencia sobre el tercero en la mayoría de los casos de la bibliografía consultada, siendo reconocida como una de las causas potenciales de metatarsalgias. Los procesos biomecánicos anómalos que sobrecargan las estructuras de antepie pueden provocar una inflamación de la cápsula sinovial que delimita la articulación, disminuyendo progresivamente la movilidad del dedo y agravando la progresión de la patología.

Palabras clave: sinovitis; articulación metatarsofalángica; cápsula articular; plato glenoideo; subluxación.

\begin{abstract}
In podiatric clinics is very common to find inflamatory process in metatarsophalangeal joint capsule, plantar plate and collateral ligaments damage, but it is not clearly recognized. Many authors hipotetized with joint instability of multiple aetiology and his concomitant evolution in different stages with own joint disease. This pathology has more incidence in second metatarsophalangeal joint than third and others and it is a common etiology of metatarsal pain. Bad biomechanics alters forefoot function and can produce overload in capsular joint, decreasing mobility and getting worse the pathology.
\end{abstract}

Key words: synovitis; metatarsophalangeal joint; joint capsule; plato glenoid; subluxation.

Referencia normalizada: Sánchez Gómez R, González Gutiérrez R, López López D, Losa Iglesias M, Soriano Medrano A, Morales Ponce A. Sinovitis de la segunda articulación metatarsofalángica: actualización. Rev. Int. Cienc. Podol. 2016; 10(2): 95-105.

Sumario: Introducción. Histología y funcionalidad. Etiopatogenia. Clínica. Modalidades de diagnóstico. Diagnóstico diferencial. Tratamiento. Conclusiones. Bibliografía. 


\section{INTRODUCCIÓN}

En los años 70 algunos clínicos ya empezaron a valorar la existencia de un proceso inflamatorio a nivel de la articulación metatarsofalángica del segundo dedo que tenía diferentes estadíos de evolución. Pero no fue hasta la década de los años 90 cuando autores como Trepman, Yeo y otros $^{1-8,11}$ agruparon una serie de signos y síntomas asociados a esa patología inflamatoria de la segunda articulación metatarsofalángica del pie con el nombre de sindrome de predislocación. Este es un término acuñado del inglés que define una entidad clínica según la cual se puede producir una inflamación, una subluxación o una luxación completa de la articulación metatarsofalángica de los dedos, siendo la prevalencia en el segundo dedo mayor que en el tercero y a su vez mayor que en el resto ${ }^{1-8}$. Es una patología que se presenta en numerosas metatarsalgias y que requiere por tanto una minuciosa historia clínica para poder definir ya no solo la etiología causante de la inflamación y poder actuar en consecuencia, si no también para averiguar a partir de la semiología en cual de esas tres etapas (inflamación, subluxación o luxación completa de la articulación) nos encontramos, ya que cada uno de ellas tiene un abordaje terapeútico diferente.

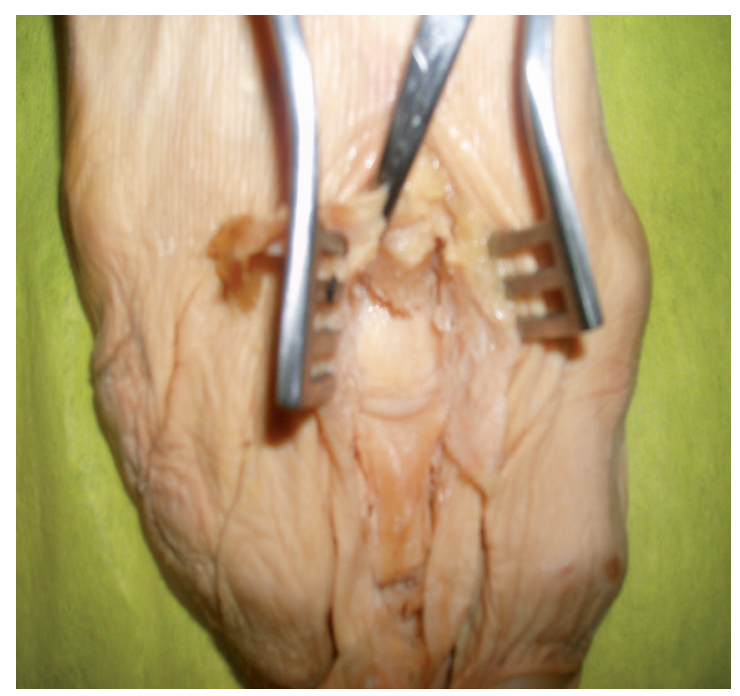

\section{HISTOLOGÍA Y FUNCIONALIDAD}

El plato plantar, placa plantar o plato glenoideo es una estructura fibrocartilaginosa que nace en el cuello distal del metatarsiano precedente y se inserta proximal en la diáfisis de la falange proximal, siempre en la región plan$\operatorname{tar}^{1,11,12}$; es una estructura cóncava por la que la cabeza metatarsal rueda en el momento del despegue del pie y además sirve de mecanismo de estabilización de músculos interóseos, lumbricales y ligamentos metatarsofalángicos (Figuras 1 y 2).

\section{ETIOPATOGENIA}

Según la mayoría de los autores consultados, la etiología de esta entidad clínica hay que centrarla en la inestabilidad de la AMTF.

Existen estudios ${ }^{7,8}$ en los que, tras el cribaje de alteraciones sistémicas como el factor reumatoideo, anticuerpos antinucleares o Lyme titer que pudieran justificar el proceso inflamatorio, se evidenció la inflamación de la II AMTF. Los estudios realizados llevaron a los autores a la hipótesis de que la hiperextensión forzada de dichas articulaciones provocaría una elongación de la aponeurosis plantar y de la cápsula

Figuras 1 y 2. Plato glenoideo (cabezas de flecha) tras la resección de las estructuras dorsales. 
articular. Una elongación de la cápsula articular y de su expansión a la falange proximal del dedo, junto con la de la aponeurosis plantar, son motivo suficiente para que la articulación quede inestable y por consiguiente, pierda funcionalidad. El desequilibrio muscular entre los músculos intrínsecos y extrínsecos que ahora actúan con diferentes brazos de palanca por esa falta de estabilidad articular puede ser el origen de la subluxación / luxación de las articulaciones metatarsofalángicas, dado que el dedo, a través de su falange proximal, queda desalineado en flexión dorsal (FD) condicionando al plato glenoideo, que también empezará a subluxarse dorsalmente. Este proceso patomecánico llevado a la cronicidad supone, según los autores, un factor desencadenante de daño articular que tiende a la fijación rígida del dedo.

Está descrito ${ }^{2,6}$ que la fascia plantar poseé inserciones accesorias en las falanges proximales, en los platos glenoideos y en los ligamentos colaterales. Se ha podido comprobar que en las disecciones cadavéricas, la aparente mal posición de la articulación metatarsofalángica implicada está asociada con la patología del plato glenoideo y los ligamentos colatera$\operatorname{les}^{2}$. Por otra parte, Coughlin atribuyó también esta circunstancia a los atletas, como consecuencia de la continua solicitación que sufren estas articulaciones durante la carrera. El mecanismo de Windlass se podría ver afectado y su disfunción afectar a la estabilidad de la falange proximal del dedo. La fascia plantar puede verse retraída por numerosas patologías estructurales del pie ${ }^{9}$. En los pies cavos la distancia entre origen e inserción de la fascia está disminuida, y el mecanismo de Windlass ${ }^{9,10}$ no es capaz equilibrar la absorción del impacto contra el suelo en el momento del apoyo completo, provocando así un exceso de tensión y el consiguiente engarramiento de los dedos menores, formando el conocida patología de dedos en garra por "flexor substitution", en la que también se ve asociado el tríceps sural. Así mismo, un retropie varo parcialmente compensado con un antepie valgo, o un retropie valgo compensado con un antepie varo son dos fenómenos patológicos de alto daño en el pie; entre las estructuras afectadas podemos destacar en ambos casos a la fascia plantar, que se ve sometida a un giro longitudinal permanente, desencadenante de un aumento de tensión en sus inserciones. Además, en el retropie valgo compensado con antepie varo, el músculo cuadrado plantar de Silvio, que estabiliza la flexión plantar del músculo flexor largo común de los dedos para acercar a estos al suelo y ganar en estabilidad en los momentos de apoyo completo, fase de propulsión y despegue, ve disminuida su distancia entre origen e inserción y no estabilizará dicho movimiento de flexión plantar, con lo que aparecerá también la garra de los dedos (flexor stabilization) ${ }^{9}$. Este engarramiento unido a la poca disponibilidad de la fascia comprometerán al plato glenoideo y a los ligamentos colaterales de las falanges, que son las principales estructuras responsables de guardar la estabilidad de las articulaciones metatarsofalángicas de los dedos. Otros autores ${ }^{1,3,4-8}$ se desmarcan del planteamiento de Coughlin y hablan de la deformidad del hallux abductus valgus, consecuente de una alteración biomecánica de la primera articulación metatarsofalángica, ya que afirman que, a parte de provocar el desplazamiento del $2^{\circ}$ dedo hacia dorsal por falta de espacio físico, forzando su posición patológica, la transmisión de cargas en el despegue por el primer radio pierde protagonismo y es el segundo quién debe soportar durante mas tiempo mayor número de cargas, aumentando el momento de fuerzas sobre su articulación metatarsofalángica, la cual no está habilitada para tal objetivo.

De manera similar por la que se puede sobrecargar la articulación metatarsofalángica del segundo dedo es por la alteración estructural en longitud del primer metatarsiano ${ }^{6,8-13}$, esto es, que sea mas corto que el segundo, ya que esta condición implica una serie cambios funcionales en dinámica muy parecidos a los del hallux abductus valgus que pueden llevar consigo la sobrecarga de las estructuras metatarsofalángicas del segundo dedo. Hablamos de un primer metatarsiano mas corto cuando en la parábola metatarsal, este es mas corto que el tercero ${ }^{1,9,10}$. La disminución en longitud (descrita por primera vez por Morton en 1935) implica una redistribución de cargas lejos del primer metatarsiano a los metatarsianos vecinos, concretamente al segundo, dando en algunos casos lo conocido con el nombre de síndrome de Morton, estando presente cuando exista un 
primer metatarsiano acortado e hipermóvil y una diáfisis del segundo metatarsiano engrosada, como consecuencia de la sobrecarga que se está produciendo. La segunda cabeza metatarsal y la articulación se verán comprometidas por un aumento de fuerzas excesivo en el momento del despegue, ya que el pie pivotará sobre ellas y no sobre el primer metatarsiano en esta fase de la marcha.

Así mismo, la insuficiencia del primer radio causada por síndromes de hipermovilidad generalizada, como el síndrome de Ehlers-Danlos o el síndrome de Marfan, también implicarán la sobrecarga de las articulaciones metatarsofalángicas contiguas ${ }^{8}$.

Una alteración en la longitud del primer o segundo metatarsiano pueden desencadenar mecanismos compensatorios a nivel de la articulación subastragalina ${ }^{10,13}$. Un primer metatarsiano demasiado corto tiene como fenómeno estabilizador una pronación de la articulación mediotarsiana, o en su defecto de la articulación subtalar, para intentar dar estabilidad al pie en la fase de apoyo completo y despegue. Una excesiva pronación de la articulación subastragalina ocasiona un cambio en el brazo de palanca del músculo peroneo lateral $\operatorname{largo} 0^{9-13}$, que consecuentemente no podrá estabilizar en flexión plantar al primer radio en el momento del despegue. Se crea entonces un estado de hipermovilidad del primer radio, con desviación en flexión dorsal y adducción del primer metatarsiano. Consiguiente a esto, aparecerá una pérdida funcional para la flexión dorsal del primer metatarsiano, que ya está colocado en esa posición, existirá un aumento de las cargas en el segundo radio, implicando así a las estructuras encargadas de mantener su estabilidad como son la cápsula sinovial, plato glenoideo y los ligamentos colaterales. Estas estructuras reaccionarán primero inflamándose y rompiéndose después, llevando al dedo a una posición de flotante fl, $^{1,4,6,8-16}$

\section{CLÍNICA}

Es importante realizar una buena historia clínica para determinar aquellos hábitos de vida del paciente que nos puedan guiar hacia una posible etiología de la enfermedad y de esta manera tratarla de manera correcta. La clínica de esta patología va a ser variada dependiendo del estadío en el que nos encontremos. En los artículos consultados se hace evidente la progresión de la entidad clínica conforme se va dañando la articulación como consecuencia del proceso de sobrecarga al que se ve sometida. De entre todos los datos consultados podemos destacar varios aspectos clínicos comunes como son la inflamación de la articulación, la disminución del espacio articular (visible en radiografías dorsoplantares), el dolor y el desplazamiento de la falange proximal con respecto al metatarsiano. Según Coughlin ${ }^{3}$, la sinovitis de la segunda articulación metatarsofalángica a menudo se caracteriza por una inflamación localizada alrededor de dicha articulación.

Las primeras fases de la patología se caracterizarán porque la inflamación y el resto de signos característicos son irreconocibles. Estarán presentes el eritema y el edema de la articulación y estos se extenderán hasta la base del dedo, así como dolor a la palpación, con disminución del rango de movimiento, sobre todo a la extensión. El desarrollo de la inestabilidad articular es insidioso y no está presente inicialmente, pero puede causar marcha antiálgica en los procesos crónicos ${ }^{3,8}$. Los pacientes notarán un dolor focal crónico en el aspecto plantar $^{8}$, que disminuye en reposo y que durante la marcha se asemeja al de "andar sobre una piedrecilla". En estadíos mas avanzados de la deformidad, la sinovitis articular estará claramente presente y la cápsula articular comenzará a distenderse.

De este modo la evolución de la patología se hace inminente si no se pone tratamiento, ya que estos elementos que están manteniendo la congruencia articular se están elongando y dejan de ser funcionales. Los tendones de los músculos tendrán ahora diferentes brazos de palanca porque la falange proximal se colocará dorsal con respecto al metatarsiano (el plato glenoideo no frena dicha flexión dorsal $\mathrm{y}$ los ligamentos colaterales tampoco detienen los movimientos en el plano transverso porque ambas estructuras están elongadas) así que el dedo afectado iniciará un proceso de engarramiento, denominado por Coughlin como floating toe deformity o crossover toe deformity, en el que el dedo terminará por quedar flotante, 
sin entrar en contacto con el suelo y que evolucionará hacia una deformidad rígida irreducible, ya que esa elongación que se localiza en el plato glenoideo y ligamentos colaterales es tal que estos llegan a romperse.

Coughlin ${ }^{1,3}$ evidenció la presencia de esta deformidad y la definió como una subluxación que se da en el plano sagital y transverso y en la que normalmente el segundo dedo se superpone por encima del hallux. Su flexibilidad está presente en estática y se corrige cuando el pie está relajado o en equino. Es raro encontrar presencia de lesión hiperqueratósica en el aspecto dorsal de la articulación interfalángica del dedo afectado hasta no estar en estadíos cercanos a la cronicidad, aunque si es posible notar cierto eritema ${ }^{8,13-16}$ (Tabla 1$)$.

Además del diagnóstico de visu, está descrita una prueba que es de gran utilidad para comprobar el nivel de funcionalidad del plato glenoideo y por tanto en nivel de desplazamiento de la articulación, así como la existencia de dolor. Thompson y Hamilton ${ }^{3}$ han descrito un test de traslación del dedo o test manual de estrés vertical (Figura 3), definido también por Lachman como test digital, con el que se diferencian diversos grados de desplazamiento vertical de la falange proximal con respecto al metatarsiano, en el plano sagital.

En esta prueba se debe colocar el pie del paciente en una posición neutra y se estabilizará el metatarsiano en el plano sagital. El dedo índice y pulgar de la otra mano del explorador se colocarán dorsal y plantar a la base de la falange y se ejercerá una fuerza vertical dorsal en el plano sagital. El test se considerará positivo cuando el desplazamiento dorsal de la falange (no confundir con dorsiflexión) con respecto al metatarsiano sea igual o mayor a $2 \mathrm{~mm}$. De esta manera se pueden diferenciar cuatro estadíos según el desplazamiento que aparezca en la articulación. En el grado 0, no existe laxitud para el desplazamiento dorsal. En el grado 1, la base de la falange proximal puede desplazarse verticalmente, subluxándose del metatarsiano, pero sin llegar a luxarse. En el grado 2 la base de la falange se puede luxar, pero este desplazamiento también se puede reducir manualmen-

Tabla 1. Estadíos de la sinovitis.

ESTADÍOS DE LA SINOVITIS

\begin{tabular}{lllll} 
& $\begin{array}{l}\text { EDEMA / } \\
\text { INFLAMACIÓN }\end{array}$ & DOLOR & DESVIACIÓN & $\begin{array}{l}\text { TEST ESTRÉS } \\
\text { VERTICAL }\end{array}$ \\
ESTADÍO 1 & $\mathrm{Si}$ & $\mathrm{Si}$ & $\mathrm{No}$ & Positivo. Grado 0-1 \\
ESTADÍO 2 & $\mathrm{Si}$ & $\mathrm{Si}$ & $\mathrm{Si}$ & Positivo. Grado2 \\
ESTADÍO 3 & $\mathrm{Si}$ & $\mathrm{Si}$ & $\mathrm{Si}$ & Positivo. Grado2-3 \\
\hline
\end{tabular}

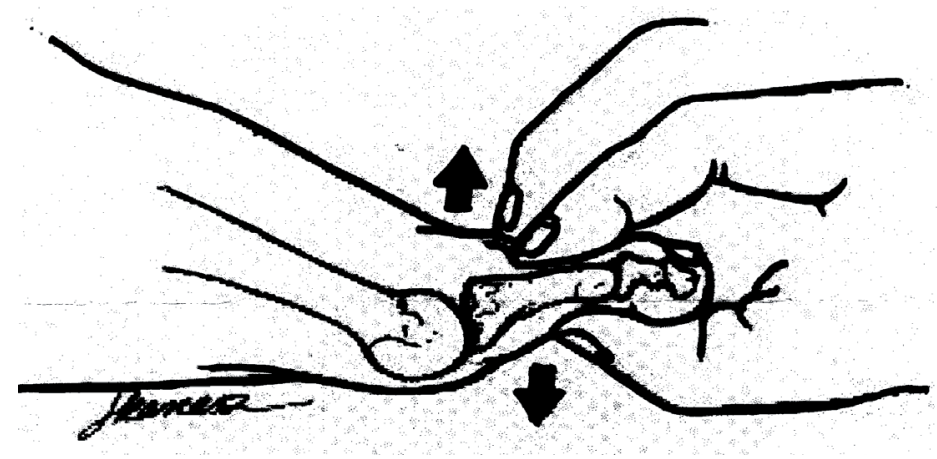

Figura 3. Test de estrés vertical. 
te. En el grado 3, la base de la falange proximal está fija en una posición luxada. Generalmente, el dolor se reproduce con esta maniobra de estrés vertical. (Tabla 1).

\section{MODALIDADES DE DIAGNÓSTICO}

Para llegar a un juicio diagnóstico certero es necesario llevar a cabo una serie de pruebas complementarias que nos corroboren nuestra hipótesis clínica.

Es útil la realización de un examen radiográfico exhaustivo de la articulación metatarsofalángica afectada para asegurar nuestras sospechas diagnósticas. La radiografía dorsoplantar (RX DP) en carga es el disparo ideal para observar el desplazamiento de la falange sobre el plano transverso (en abducción o en adducción) y las posibles rotaciones que presente, así como la disminución del espacio articular y la congruencia entre las carillas articulares. Pondremos especial énfasis en aquellos hallazgos radiográficos que nos evidencien desplazamiento dorsal y lateral o medial de la falange proximal con respecto al metatarsiano, esto es, disminución del espacio articular. El espacio articular estará disminuido desde el primer estadío. En los estadíos posteriores la subluxación / luxación en el plano sagital y / o transverso se comprobarán de igual modo en sendas radiografías laterales y dorsoplantares. Los signos radiográficos (Tabla 2) también son importantes para determinar la longitud de los metatarsianos (evidenciaremos posible etiología) y la presencia de cambios artríticos en la articulación que nos darán a conocer el daño existente y por tanto la evolución de la patología $^{1,3,8-16}$. También está descrita ${ }^{8}$ una prueba de estrés vertical radiográfica en la que se visualice este desplazamiento de la falange.

Cuando no existe evidencia clínica clara de esta patología entran en juego otras pruebas mas precisas tales como una artrografía ${ }^{8}$ o resonancias magnéticas (RM).

La artrografía es una prueba que se lleva a cabo con la inyección de 0.5 a $1 \mathrm{~mL}$ de material de contraste yodado; la punción se realiza dorsalmente a la articulación y bajo fluoroscopia, para comprobar el estado de esa articulación metatarsofalángica. El escáner de hueso trifásico nos muestra el proceso inflamatorio, gracias al diferente patrón de captación del contraste según la zona inflamada.

Por otra parte, una imagen de resonancia magnética puede mostrar el desplazamiento articular y sobre todo los signos inflamatorios o de lesión del plato glenoideo y ligamentos colaterales en imágenes potenciadas en T1 y T2. En T1 el plato glenoideo aparecerá como una estructura suave de baja señal que se deberá diferenciar del tendón flexor subyacente. Una fractura del plato glenoideo aparecerá como una señal de intensidad elevada (Figura 4) y el área de ruptura como una señal isointensa con fluido sinovial y articular. La sinovitis de

Tabla 2. Resumen del grado de severidad según el test de estrés vertical.

GRADOS DEL TEST DE ESTRÉS VERTICAL Y RELACIÓN CON RX

\begin{tabular}{llll} 
& REDUCTIBILIDAD & DESVIACIÓN & $\begin{array}{l}\text { ESPACIO ARTICULAR } \\
\text { en RX }\end{array}$ \\
GRADO 0 & No existe desviación & No & Intacto \\
\hline GRADO 1 & Subluxación Reductible & Dorsal & Disminuido \\
GRADO 2 & $\begin{array}{l}\text { Luxación } \\
\text { Reductible }\end{array}$ & Dorsal y lateral $/$ medial & Disminuido \\
\hline GRADO 3 & Luxación Irreductible & Dorsal y lateral $/$ medial & Muy disminuido \\
\hline
\end{tabular}




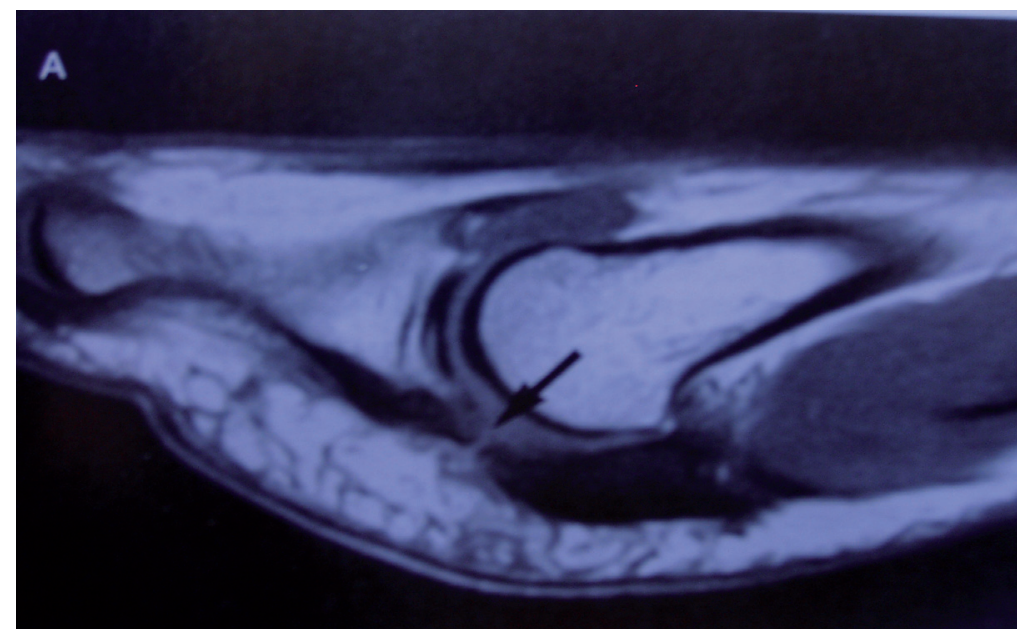

Figura 4. Ruptura del plato glenoideo (flecha) en T1 corte sagital RM II AMTF.

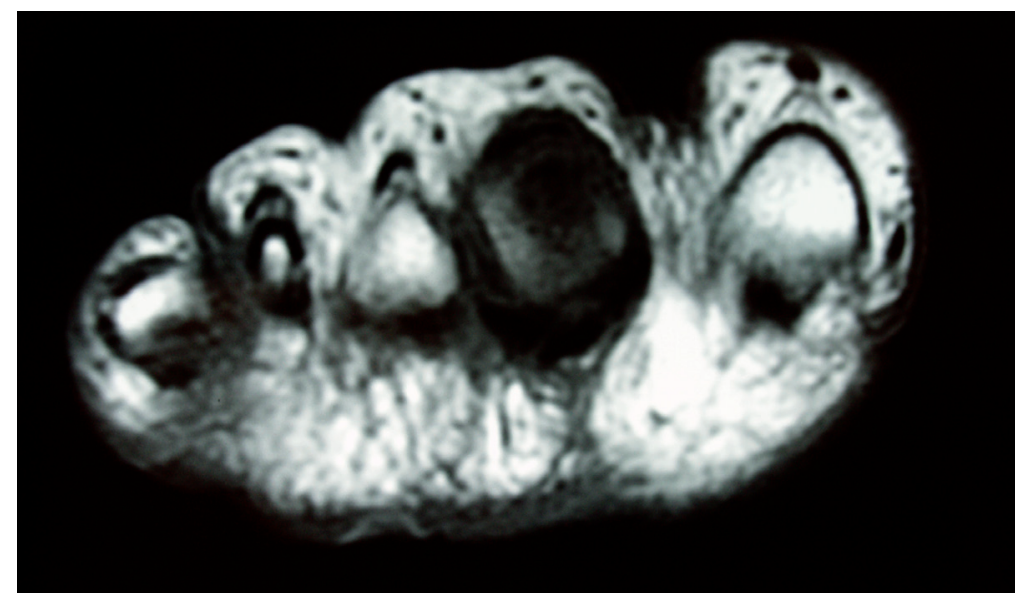

Figura 5.- RM en T1; corte coronal AMTF pie derecho. Sinovitis II AMTF. Obsérvese la inflamación localizada a nivel del segundo dedo.

la cápsula articular o la distensión o ruptura del plato glenoideo y de la cápsula sinovial son signos fácilmente identificables en una resonancia magnética ${ }^{8}$ (Figura 5).

A modo de resumen podemos decir que los diferentes autores consultados dividen a la sinovitis de la segunda articulación metatarsofalángica en tres estadíos de evolución según la clínica y hallazgos radiológicos: $3,6,8,11,12$

- Estadío 1: existe un edema plantar leve en la articulación metatarsofalángica.
Hay sensibilidad dolorosa a la manipulación de la articulación. No se aprecia desviación anatómica de la falange proximal con respecto al metatarsiano. El test de estrés vertical es positivo, grado 1 .

- Estadío 2: el edema es moderado ; existe una desviación del dedo apreciable clínica y radiológicamente. El dedo afectado no suele apoyar en el suelo. El test de estrés vertical es positivo, grado 2 . 
- Estadío 3: el edema es moderado en todo el contorno de la articulación metatarsofalángica, no solo en el aspecto dorsal o plantar, sino también en el medial y lateral, y se extiende por la base del dedo, e incluso sigue distal. Está presente una desviación pronunciada, con posible subluxación / luxación que si sigue progresando dará como resultado final un dedo supradductus con o sin deformidad de hallux abducto valgus concomitante. (Tabla 2 ).

\section{DIAGNÓSTICO DIFERENCIAL}

Es importante saber diferenciar entre los síntomas dolorosos producidos por un neuroma de Morton y los signos inflamatorios de una sinovitis de la articulación metatarsofalángica, en cualquiera de sus tres estadíos, secundarios al daño de la cápsula articular. Un dolor agudo en el aspecto plantar de la articulación (plato glenoideo) nos puede indicar presencia de inflamación de la cápsula articular. La administración de anestesia para el bloqueo de la rama del nervio plantar externo puede servir de diagnóstico diferencial de neuroma de Morton ${ }^{6}$.

Histológicamente es importante resaltar que la sinovitis se refiere a un proceso inflamatorio de la membrana sinovial que integra la cápsula articular y la capsulitis se usa para describir genéricamente la inflamación de la membrana y cápsula articular, aunque clínicamente es muy difícil diferenciarlas ${ }^{1,3}$.

Así mismo, también hay que diferenciar entre estas dos entidades clínicas y las bursitis intermetatarsales; estas son un proceso inflamatorio de la bolsa serosa que se encuentra entre las cabezas metatarsales. Los adipocitos contenidos en las celdas plantares se inflaman y provocan el dolor ${ }^{16}$.

Tambien puede ocurrir que exista una migración de la grasa subcapital secundaria a la luxación metatarsolfalángica, El almohadillado graso plantar pierde eficacia amortiguadora encontrándose disminuida la capacidad de absorción de presión, que recae directamente sobre la cabeza metatarsal, dando una de las etiologías de la metatarsalgia ${ }^{15-17}$.

\section{TRATAMIENTO}

Podemos diferenciar dos tipos de abordaje terapéutico ante la sinovitis: conservador y quirúrgico.

\section{Tratamiento Conservador}

Es un tratamiento conjunto en el que podemos combinar diferentes elementos de entre los que podemos destacar la administración de AINE's (anti-inflamatorios no esteroideos), infiltración de corticoesteroides, colocación de soportes plantares, modificaciones en el calzado y colocación de vendajes funcionales en la articulación metatarsofalángica afectada. Dependerá del dolor, grado de la deformidad y limitaciones del paciente. Será primordial reducir la carga en el antepié en el momento del despegue, para evitar la presión en la articulación afectada retrasando así la progresión de la patología hacia estadíos mas avanzados, ya que precisamente es el aumento de fuerzas que soporta el segundo metatarsiano lo que va a provocar el compromiso articular y el consiguiente daño de las partes blandas.

Por ello, se cual sea el grado de deformidad, se hace necesaria la colocación de una órtesis plantar que compense en la medida de lo posible la inestabilidad articular y aborde el porqué del aumento de fuerzas en el antepie. Tras el estudio del pie del paciente y la elección de los elementos necesarios para la compensación de la inestabilidad, la pieza que se describe es un elemento de descarga bajo la zona dañada.

La Administración de AINE's está descrita por Mann y $\mathrm{Mizel}^{3}$. Es un tratamiento sintomático en aquellos procesos álgicos inflamatorios de pequeño calibre, en los que con la colocación de una órtesis plantar y/o vendaje funcional, el problema tiene buena evolución. Los autores hablan de la administración de medicación anti-inflamatoria para disminuir esa inflamación, aunque comentan la necesidad de actuar con otros tratamientos, como por ejemplo modificaciones del calzado, para lograr una remisión completa de la patología. Así mismo, Coughlin también usó en su estudio ${ }^{1,6}$ antiinflamatorios orales para la reducción del dolor y el receso del hinchazón.

Otra opción terapéutica a tener en cuenta es la infiltración de corticoides. Su administra- 
ción busca el alivio sintomático del dolor (y la inflamación) y recuperar la capacidad para caminar sin molestias. Se recurre a este procedimiento terapéutico cuando la sintomatología dolorosa no ha remitido ni con el soporte plantar ni con el reposo. Está descrita como una inyección vía dorsal y evitando los tendones extensores, a nivel del espacio intermetatarsal afectado, realizando la flexión plantar de los dedos ${ }^{7}$ para la apertura del espacio intermetatarsal. Se han descrito alteraciones en los tejidos ligamentosos y destrucción de los fibrocitos ${ }^{8} \mathrm{y}$ por ello el clínico debe utilizar la dosis efectiva más pequeña de sal fosfórica; hay que tener presente los efectos secundarios indeseados de la administración de corticoides, entre los que podemos destacar el daño que producen sobre las fibras de colágeno. El plato glenoideo posee fibras de colágeno tipo II, y los ligamentos colaterales también están formados por estructuras de colágeno y por todo ello una articulación se inyectará como máximo una vez de uno a tres meses y no mas de dos a tres veces en un periodo de doce meses. Son diferentes los autores que han seguido la pauta de administrar corticoesteroides. Treppman y $\mathrm{Yeo}^{7}$ administraron $1 \mathrm{ml}$ de dexametasona, $4 \mathrm{mg} / \mathrm{ml}$, o $1 \mathrm{ml}$ de triamcinolona, $40 \mathrm{mg} / \mathrm{ml}$, mezclado con $1 \%$ de lidocaína $\left(0^{\prime} 5-1 \mathrm{ml}\right)$ y $0^{\prime} 5 \%$ de bupivacaína $\left(0^{\prime} 5-1 \mathrm{ml}\right)$ a 12 de sus 13 pacientes con sinovitis de la segunda articulación metatarsofalángica. El restante optó por la administración oral de $400 \mathrm{mg}$ de Ibuprofeno. Los resultados que obtuvieron, en conjunción con el tratamiento de modificación del calzado, fueron satisfactorios. Mizel y Treppman ${ }^{3}$ administraron también fármacos esteroideos a 30 pacientes, obteniendo un $70 \%$ de buenos resultados, es decir, cursando con la reducción o desaparición de la sintomatología inflamatoria y dolorosa en ellos. Mizel y James ${ }^{5}$ realizaron un estudio de 4 años y medio de duración a 13 pacientes, 11 mujeres y 2 hombres, con sinovitis de la segunda articulación metatarsofalángica, sin desviación lateral y hallux abductus valgus (HAV) asociado. Procedieron a la inyección intraarticular de dexametasona, bupivacaína al 0'5 \% y lidocaína al 2\% sin epinefrina, en volúmenes iguales. Este tratamiento lo combinaron con el uso de un calzado modificado y de los 14 pies estudiados, este tratamiento conservador no dio resultado tan solo en uno de ellos, al cual hubo que intervenir quirúrgicamente. Hay que hablar así mismo de la administración oral de esteroides ${ }^{8}$. La principal ventaja de esta terapia es el evitar la siempre traumática experiencia que la infiltración le causa al paciente. Las pautas de dosificación encontradas en la bibliografía son de 60 a 10 $\mathrm{mg}$ con aumentos de $10 \mathrm{mg}$ de prednisona, administrada en 21 tabletas de $10 \mathrm{mg}$ cada una.

Pero si lo que se quiere es frenar la progresión de la deformidad en el plano sagital Coughlin y otros autores ${ }^{1,6,7,8}$ observaron buen resultado en el abordaje de un dedo flotante moderado con la colocación de un vendaje; este consistía en estabilizar la falange proximal en una posición de plantarflexión gracias a la colocación de las tiras en forma de corbata. También usaron una tablilla esponjosa plantar para fijar a esta falange en la posición requerida. Pero el uso del vendaje lleva consigo el inconveniente de que se puede crear edema y una posible ulceración del dedo $^{6,7,8}$, ya que el tiempo necesario para que se creen puentes fibróticos que le reporten estabilidad a la articulación afectada suele ser de varios meses. El uso de esa almohadilla metatarsiana puede evitarnos este trastorno. Una temprana interrupción del entablillado puede dar como resultado una subluxación progresiva de la articulación, con lo que es preciso llevar un control estricto del paciente para que no exista posibilidad de empeoramiento de la patología.

Se ha comentado la posibilidad de realizar una modificación del calzado. Dicha opción es valorable siempre, independientemente de cual sea el estadío en el que nos encontremos. Consistiría en crear un zapato extra-hondo para acomodar la deformidad digital combinado con una suela rocker bottom, también conocida con el nombre de balancín ${ }^{6,7,8,12}$, situado desde la zona mas distal de la suela del zapato hasta el enfranque, muriendo a cero en proximal y distal, con máxima altura de cinco milímetros en el punto medio entre estos puntos proximal y distal, para reducir las fuerzas propulsivas en la articulación metatarsofalángica dañada, realizándose por esta zona el despegue y no por la dorsiflexión de las falanges con respecto a los metatarsianos. Adicionalmente, algunos auto$\mathrm{res}^{8}$ usan una terapia física agresiva con manipulación de los tejidos contraídos dorsalmente y así reducir la inflamación. 


\section{Tratamiento quirúrgico}

Una vez se ha agotado la vía conservadora, se procederá a la intervención quirúrgica, consistente en la reducción de la deformidad en el plano sagital y/o transverso, con la realización de artroplastia, artrodesis, liberación de la AMTF y/o la transposición de los tendones flexores y reparación de la cápsula, plato glenoideo y estructuras adyacentes dañadas ${ }^{1,2,3,6,8,14-17}$, dependiendo del grado lesivo en el que nos encontremos.

\section{CONCLUSIONES}

La sinovitis o inflamación de la cápsula articular de la segunda articulación metatarsofalángica es una causa de dolor en el antepié y por tanto un motivo mas de consulta podológica. Es importante conocer bien la etiopatogenia para relacionar los signos y síntomas descritos por el paciente con el grado de deformidad en el que nos encontramos.

La función que realizan el plato glenoideo y los ligamentos colaterales es de máxima importancia para mantener la integridad de las articulaciones metatarsofalángicas menores en estática y en dinámica. El aumento de fuerzas en la articulación es el motivo fundamental por el que se produce la sinovitis y la consecuente inestabilidad articular. El daño del plato glenoideo y los ligamentos colaterales es el resultado de una inestabilidad mantenida en el tiempo y por ello se hace necesaria una redistribución de las cargas que recaen sobre esa articulación en primer lugar y un buen equilibrio muscular intrínsecoextrínseco de los músculos flexores y extensores en segundo lugar para salvaguardar la integridad morfo-funcional de la articulación. Esta segunda premisa se contempla quirúrgicamente solo cuando las opciones conservadoras de redistribución de cargas no han dado resultado.

\section{BIBLIOGRAFÍA}

1. Coughlin MJ. Second Metatarsophalangeal Joint Instability in the Athlete. Foot and Ankle. 1993; 14(6): 309-319.

2. Deland JT, Kyung-Tai Lee. Anatomy of the plantar Plate and Its Attachments in the Lesser Metatarsal Phalangeal Joint. Foot and Ankle International. 1995; 16(8): 480-486.

3. Fortin PT, Myerson MS. Second Metatarsophalangeal Joint Instability. Foot and Ankle International. 1995;16 (5): 306-313

4. Mann RA, Mizel MS. Monoarticular Nontraumatic Synovitis of the Metatarsophalangeal Joint: A new Diagnosis?. Foot and Ankle. 1985; 6(1): 18-21.

5. Mizel MS, Michelson JD. Nonsurgical Treatment of Monoarticular Nontraumatic Synovitis of the Second Metatarsophalangeal Joint. Foot and Ankle International.1997; 18 (7): 424-426.

6. Mendicino RW, FACFAS. Predislocation Sindrome: A review and Retrospective Análisis of Eight Patients. The Journal of Foot and Ankle Surgery. 2001; 40(4):214-224.

7. Trepman E, Yeo SJ. Nonoperative Treatment of Metatarsophalangeal Joint Synovitis. Foot and Ankle International. 1995; 16(12): 771-777.

8. Yu GV, Judge MS, Hudson JR, Seidelmann FE. Predislocation syndrome. Progressive subluxation/ dislocation of the lesser metatarsophalangeal joint. J Am Podiatr Med Assoc. 2002 Apr;92(4):182-99.

9. Green DR. Extensor and Flexor Substitution - Digital Surgery. 17. Second Annual Metropolitan Hospital Surgical Symposium Joint Disease and Limb Salvage, March 1983.

10. Fuller EA. The Windlass Mechanism of the foot. A Mechanical Model to Explain Pathology. Journal of the American Podiatric Medical Association. 2000; 90(1) : 35-46.

11. Yao L, Do HM, Cracchiolo A, Farahani K. Plantar plate of the foot: findings on conventional arthrography and MR imaging. Australasian Journal of Podiatric Medicine 2003; 37(2) : 43-46.

12. Gerard VYu, Judge MS, Hudson JR, Seidelmann FE. Predislocation Syndrome Progressive Subluxation/Dislocation of the Lesser Metatarsophalangeal Joint . American Journal of Roentgenology, Vol 163, 641-644, Copyright (C) 1994 by American Roentgen Ray Society.

13. Munuera Martín PV. El primer radio. Biomecánica y Ortopodología. Santander: Exa Editores SL; 2009. 
14. Molloy A, Shariff R. Mallet toe deformity.Foot Ankle Clin. 2011 Dec;16(4):537-46.

15. Smith BW, Coughlin MJ. Disorders of the lesser toes. Sports Med Arthrosc. 2009 Sep;17(3):167-74.

16. Schrier JC, Verheyen CC, Louwerens JW. Definitions of hammer toe and claw toe: an evaluation of the literature. J Am Podiatr Med Assoc. 2009 May-Jun;99(3):194-7.

17. Shirzad K, Kiesau CD, DeOrio JK, Parekh SG. Lesser toe deformities. J Am Acad Orthop Surg. 2011 Aug;19(8):505-14 